\title{
Searching for Ultracool Companions with NICI
}

\author{
C. Ftaclas and E. L. Martín \\ Institute for Astronomy, Univ. of Hawaii, 2680 Woodlawn Dr., \\ Honolulu, HI 96822 \\ D. Toomey
}

IfA-Hilo and Mauna Kea Infrared

\begin{abstract}
We present to the brown dwarf community the Near Infrared Coronographic Imager (NICI), which is being built for installation in 2005 on the Gemini South Telescope. NICI's dual channel architecture and dedicated AO system are designed to permit deep circumstellar discrimination of faint sources where some spectral key can be used to get a simultaneous estimate of the background. One of the principal science goals of NICI is to search for ultracool companions to stars and brown dwarfs.
\end{abstract}

\section{Introduction}

Adaptive optics (AO) systems increase the dynamical range of astronomical observations making it possible to detect ultracool dwarf companions to solar-type stars such as those reported by Liu et al. (2002) and Potter et al. (2002). Both of them were first detected with Hokupa'a on Gemini, and follow-up spectroscopy was secured with Keck/NIRSPEC-AO. Since the number of solar-type stars observed with $\mathrm{AO}$ systems in very large ground-based telescopes is still small $(<50)$, these two companions suggest that brown dwarfs may be relatively common around stars at separations larger than $10 \mathrm{AU}$. On the other hand, it is well known that brown dwarfs are rare within $5 \mathrm{AU}$ of solar-type stars (Vogt et al. 2002). A dependency of the frequency of brown dwarf companions with orbital separation from the star is explained by some models of orbital migration in circumstellar disks (see Armitage \& Bonnell, this volume).

The high spatial resolution provided by $\mathrm{AO}$ allows to resolve close binaries. Ultracool dwarfs can be observed with conventional AO systems if they have a bright star within about 30 arcminutes, but Hokupa'a has demostrated that the brightest dwarfs can be observed directly. Several tight binaries have been discovered using AO on the Keck and Gemini telescopes (Martín et al. 2000; Close et al. 2002). All of them have separations between 1 and 15 AU. The tighest of them has been followed up with Keck/AO and an orbit has been derived by Lane et al. (2001). These are the first dynamical masses obtained for brown dwarfs. 
In this paper we briefly present an intrument that has been designed specifically for high contrast imaging using extreme AO. One of the main science goals of this instrument is the search for ultracool companions. A custom-made curvature AO system will allow guiding on ultracool dwarfs, and hence searching for planets around brown dwarfs.

\section{The NICI advantage}

The Near Infrared Coronagraphic Imager for Gemini South (NICI) is a dual beam coronagraphic camera operating over the 1.0 to 5.5 micrometer wavelength range with a dedicated 85 -element curvature adaptive optics system. All aspects of the instrument were optimized for coronagraphic work meaning that there are a minimum number of optical elements and only the dewar window is refractive. Prior to the instrument focus, a dichroic splits the beam so that the transmitted visible light used for wave front sensing is sent to the wave front sensor on the other side of the bench. Although it is more difficult to build a dichroic that transmits the visible light, this choice permits operation at thermal wavelengths. At the instrument focus there are options for selecting one of several coronagraphic occulting masks. In a coronagraph it is desirable to minimize the number of optical elements before the focal plane mask to reduced scattered light, but AO correction must take place before the occulting mask because on an 8 meter telescope without correction the image will be so degraded that the mask will have to be too large. The goal for image quality at the detectors is $50 \%$ Strehl at K (2.2 microns). This is based on an error budget that includes AO performance according to present Gemini models and a nominal telescope performance. With this performance the image core will be about 0.06 arcseconds and will be sampled with about 3.5 pixels across the core. NICI is unique in that it has been designed from the ground up to be a coronagraphic imager and very few compromises have been made in order to support more general astronomical applications. This design philosophy is what led to the decision to include a dedicated AO system rather than try to work with a facility system. More information about NICI is given by Toomey \& Ftaclas (2003).

\section{References}

Close, L. M., et al. 2002, ApJ, 567, L53

Lane, B. F., Zapatero Osorio, M. R., Britton, M. C., Martín, E. L., \& Kulkarni, S. R. 2001, ApJ, 560, 390

Liu, M. C. et al. 2002, ApJ, 571, 519

Martín, E. L., Koresko, C. D., Kulkarni, S. R., Lane, B. F., \& Wizinowich, P. L. 2000, ApJ, 529, L37

Potter, D., Martín, E. L., Cushing, M. C., Baudoz, P., Brandner, W., Guyon, O., \& Neuhäuser, R. 2002, ApJ, 567, L133

Toomey, D. \& Ftaclas, C. 2003, SPIE, in press

Vogt, S. S., Butler, P. R., Marcy, G. W., Fischer, D. A., Pourbaix, D., Apps, K., \& Laughlin, G. 2002, ApJ, 568, 352 